Article

\title{
On the Applicability of Linear, Axisymmetric Dynamics in Intensifying and Mature Tropical Cyclones
}

\author{
Michael T. Montgomery ${ }^{1, *}$ and Roger K. Smith ${ }^{2}$ \\ 1 Department of Meteorology, Naval Postgraduate School, Monterey, CA 93943, USA \\ 2 Meteorological Institute, Ludwig Maximilians University of Munich, 80333 Munich, Germany; \\ rogerksmith@online.de \\ * Correspondence: mtmontgo@nps.edu; Tel.: +1-831-656-2296
}

Received: 31 May 2017; Accepted: 30 November 2017; Published: 13 December 2017

\begin{abstract}
The applicability of linearized axisymmetric dynamics to the intensification and structure change of tropical cyclones is investigated. The study is motivated by recent work that presented axisymmetric solutions to the linearized, non-hydrostatic, vortex-anelastic equations of motion (the so-called 3DVPAS model). The work called into question the importance of a recently proposed nonlinear, system-scale boundary-layer spinup mechanism both in intensifying storms and in mature storms undergoing secondary eyewall formation. The issue is examined using a three-dimensional mesoscale simulation of an intensifying tropical cyclone, alongside the linear 3DVPAS model. Solutions to the linear model, for imposed eddy forcing terms derived from the mesoscale simulation, are shown to be valid only for short times $(t<1 \mathrm{~h})$ in the inner-core region of the vortex. At later times, the neglected nonlinear terms become significant and the linear results invalid. It follows that the linear results cannot be used to describe all aspects of the tropical cyclone dynamics at later times. In particular, they cannot be used (a) to dismiss the importance of the nonlinear boundary-layer spinup mechanism, nor (b) to isolate the separate effects of diabatic heating from those of friction, within the nonlinear boundary layer at least. Such separation depends on the linear superposition principle, which fails whenever nonlinearity is important. Similar caveats apply to the use of another linear model, the traditional Sawyer-Eliassen balance model. Its applicability is limited not only by linearity, but also by its assumption of strictly balanced motion. Both are incompatible with nonlinear spinup.
\end{abstract}

Keywords: tropical cyclone; hurricane; typhoon; intensification; eddy dynamics; conventional and nonlinear boundary layer spinup mechanisms

\section{Introduction}

In the past few years, there have been at least two studies of tropical cyclone dynamics using a model called "Three-Dimensional Vortex Perturbation Analysis and Simulation" (3DVPAS), a linearized numerical model designed to analyze some aspects of symmetric and asymmetric vortex dynamics, including the dynamics of tropical cyclone and tornado vortices [1,2]. The 3DVPAS model neglects all nonlinear terms involving the axisymmetric flow, but generalizes classic balance models in that it retains linear unbalanced processes including radially and vertically propagating inertia-gravity waves. The studies reported in [1,2] apply the 3DVPAS model to the evolution of an axisymmetric tropical cyclone vortex by forcing the model with azimuthally-averaged outputs from three-dimensional, non-hydrostatic numerical simulations of an intensifying tropical cyclone, or a tropical cyclone undergoing secondary eyewall formation. The averaged outputs represent heating and forces arising from eddy processes in the numerical simulations. Therefore, these studies can be seen as a revival of 
the axisymmetric "convective ring model" of tropical cyclone intensification originally proposed in [3,4], but can be seen also as a generalization thereof because the original ring model assumed axisymmetric balance dynamics.

Ref. [1] used the 3DVPAS model to diagnose the relative roles of the heat and tangential momentum forcing in the formation of a simulated secondary eyewall as predicted by the three-dimensional Weather Research and Forecasting (WRF) model. The solutions for the transverse circulation using the 3DVPAS model were found to be qualitatively similar to the transverse circulation diagnosed from the WRF model. However, the theoretical justification for the neglect of the nonlinear terms was not provided. At a minimum, one needs to verify that all the nonlinear terms, when evaluated using the linear solutions, are small in comparison to the retained linear terms.

In a more recent study, Ref. [2] used the same methodology as employed in [1] to diagnose the relative roles of the heat and tangential momentum forcing distributions in the intensification of a tropical cyclone using again the WRF model, but for another intensifying storm.

Because of the linearization built into the 3DVPAS model, this use of it cannot address the situation within the frictional boundary layer in the inner-core region of the storm where the flow is intrinsically nonlinear [5-7]. As discussed in earlier publications (e.g., [7-10]), there is an intrinsically nonlinear boundary layer spinup process, which, as discussed in [7], involves strong, non-Ekman-like nonlinearities in an essential way, with dramatic consequences such as the generation of strong supergradient winds within the boundary layer. These supergradient winds include the strongest and most destructive winds found anywhere within a mature tropical cyclone.

The inherently linear nature of the 3DVPAS model means that its use in Refs. [1,2] produces results that are misleading in two ways. The first is simply that important nonlinear terms are neglected, and the second is that, in using the results to examine the relative importance of heat and tangential momentum forcing, the linear superposition principle is tacitly assumed. That is, it is assumed that the heat and momentum forcing processes can be considered separately, with effects that are additive. The superposition principle fails as soon as nonlinearity is significant, and fails drastically when nonlinearity is strong. In particular, its use in Ref. [2] to cast doubt on the nonlinear boundary layer spinup mechanism is, in our view, unjustified.

The objectives of this paper are twofold. The first is to examine the validity of the 3DVPAS model, summarized in Section 2, for a class of axisymmetric vortex flows forced by azimuthally-averaged diabatic and frictional terms diagnosed from a state-of-the art three-dimensional mesoscale numerical model (Section 3). The specific goal in this context is to quantify the errors introduced by the neglect of the nonlinear terms in the governing equations in the inner-core region of the storm (Section 4). We show that the inner-core nonlinear terms are already significant even at an early stage of tropical cyclone intensification, much earlier than expected from the recent results of [7] who examined a later stage of tropical cyclone intensification and secondary eyewall formation. Our findings, together with those of [7], suggest that the emergence of strong nonlinearities outside the scope of 3DVPAS invalidates the use of 3DVPAS for diagnosing and interpreting complex numerical model solutions for maturing and mature tropical cyclones that possess such strong nonlinearities.

A second, and subsidiary, objective (Section 5) is to examine the use of the traditional Sawyer-Eliassen (hereafter SE) model of transverse circulations, in this same context of maturing and mature tropical cyclones. The SE model was used in [2] to cast further doubt on the existence of nonlinear spinup. However, the SE model is linear, by assumption, in the same sense as the 3DVPAS model, and is subject to additional limitations through its assumption of strictly balanced motion. Neither assumption is compatible with the nonlinear boundary-layer dynamics. For instance, when the boundary-layer inflow separates and turns upward into the eyewall, not only are the non-Ekman-like nonlinearities strong, but departures from balance can become significant in the form of axisymmetric inertia-gravity waves launched into the eyewall upflow [11]. Section 5 discusses the extent to which these limitations might be overcome in future work. Section 6 offers concluding remarks. 


\section{A Forced Axisymmetric Convective Ring Model Revisited}

The time-dependent linear vortex model 3DVPAS was developed originally to examine linear, non-hydrostatic, asymmetric wave-like disturbances and their wave, mean-flow interactions in tropical cyclones and tornado vortices [12]. The model solves for linear disturbances to a basic-state circular vortex in gradient and hydrostatic balance and has produced many insightful results for a wide class of asymmetric vortex wave and instability problems in tornado and tropical-cyclone-like vortices (e.g., [12-17]).

Follow-up work by $[17,18]$ adapted this model to solve for the azimuthal-wavenumber-zero disturbance and corresponding velocity and temperature tendencies subject to the imposed forcing of diabatic heating, and momentum sources/sinks for physically-motivated forcing distributions in either tornadoes or tropical cyclones. This is the version of the model used in [1,2], where the model configurations assume an initially zero radial and vertical flow and a zero vertical gradient of basic-state tangential velocity below a nominal height that spans a large part of the boundary layer; see assumption (1) below Equation (11).

The 3DVPAS model used here is the version described in [19]. It uses a non-standard form of the anelastic equations known as the "vortex-anelastic equations", in which the basic-state density $\rho$ and potential temperature $\theta$ are allowed to depend on radial distance $r$ as well as on altitude $z$, as appropriate for a strong vortex. For the full set of equations, the reader is referred to [19].

The subset of equations from Ref. [19] needed here, and the notation to be used, are as follows. The azimuthal-wavenumber-zero disturbance flow predicted by the 3DVPAS model is denoted by a zero subscript. The asymmetric (eddy) disturbance flow is denoted by a prime. By definition, primed quantites have zero azimuthal average. They are diagnosed from the WRF model output at hour 27 (see below). The corresponding eddy forcing terms, in Equations (6) and (10) below, are switched on at the initial time $t=0$ of the 3DVPAS model. For times $t \geq 0$, we write $v=V(r, z)+v_{0}+v^{\prime}$, where $V(r, z)$ is the frozen-in-time circular vortex tangential velocity field in gradient wind and hydrostatic balance at $t=0$ (i.e., at WRF hour 27), and $v_{0}=v_{0}(r, z, t)$, the tangential velocity disturbance that develops in response to the imposed eddy forcing and satisfies $v_{0}(r, z, 0)=0$. Similarly, we write $u=u_{0}+u^{\prime}$, where $u_{0}(r, z, t)$ is the forced radial velocity disturbance satisfying $u_{0}(r, z, 0)=0$. Similar definitions apply for the vertical velocity, pressure, and potential temperature fields. Respectively, these are $w=w_{0}(r, z, t)+w^{\prime}, p=P(r, z)+p_{0}(r, z, t)+p^{\prime}$, and $\theta=\Theta(r, z)+$ $\theta_{0}(r, z, t)+\theta^{\prime}$, where $w_{0}(r, z, 0)=0, p_{0}(r, z, 0)=0$, and $\theta_{0}(r, z, 0)=0$. The anelastic density field $\rho$ is a fixed basic-state-only quantity, $\rho=\rho(r, z)$.

With these definitions, the horizontal momentum equations become

$$
\begin{aligned}
& \frac{\partial u_{0}}{\partial t}=\bar{\xi} v_{0}-\frac{\partial}{\partial r}\left(\frac{p_{0}}{\rho}\right)+F_{r}+N_{u_{0}}, \\
& \frac{\partial v_{0}}{\partial t}=-\bar{\eta} u_{0}-w_{0} \frac{\partial V}{\partial z}+F_{\lambda}+N_{v_{0}}
\end{aligned}
$$

where $\bar{\xi}=f+2 V / r$ denotes twice the local absolute rotation rate of the basic-state vortex, and $\bar{\eta}=f+(1 / r) \partial(r V) / \partial r$ denotes its local absolute vorticity. The remaining terms are defined in Equations (5)-(8) below. The basic-state pressure field $P(r, z)$ is assumed to be in gradient wind balance with $V(r, z)$ :

$$
\frac{V^{2}}{r}+f V=\frac{1}{\rho} \frac{\partial P}{\partial r}
$$

and in hydrostatic balance with $\rho(r, z)$,

$$
\frac{\partial P}{\partial z}=-\rho g
$$


where $g$ is the gravitational acceleration. This basic-state vortex is constructed by azimuthally averaging the WRF model output, as described in the next section; see also assumption (1) below Equation (11).

The terms $F_{r}$ and $F_{\lambda}$ in Equations (1) and (2) represent the imposed forcing and are defined by

$$
\begin{gathered}
F_{r}=\overline{S G_{r}}-\overline{u^{\prime} \frac{\partial u^{\prime}}{\partial r}}-\overline{w^{\prime} \frac{\partial u^{\prime}}{\partial z}}, \\
F_{\lambda}=\overline{S G}_{\lambda}-\overline{u^{\prime} \zeta^{\prime}}-\overline{w^{\prime} \frac{\partial v^{\prime}}{\partial z}}-\overline{\frac{1}{r \rho} \frac{\partial p^{\prime}}{\partial \lambda} .} .
\end{gathered}
$$

Here an overbar denotes the azimuthal average, and, as before, the primed (eddy) quantities the deviations therefrom, with zero average; $\zeta^{\prime}$ denotes the eddy vertical vorticity $(1 / r)\left(\partial\left(r v^{\prime}\right) / \partial r-\right.$ $\left.\partial u^{\prime} / \partial \lambda\right), \lambda$ is the azimuthal angle and $S G_{\lambda}$ and $S G_{r}$ denote the subgrid-scale velocity tendencies associated with surface friction and unresolved turbulent mixing processes (modeled by diffusion) in the planetary boundary layer and vortex interior.

The terms $N_{u_{0}}$ and $N_{v_{0}}$ in Equations (1) and (2) are the nonlinear terms arising from the self-advection and curvilinear acceleration of the wavenumber-zero disturbance flow, and are defined as follows:

$$
\begin{gathered}
N_{u_{0}}=-u_{0} \frac{\partial u_{0}}{\partial r}-w_{0} \frac{\partial u_{0}}{\partial z}+\frac{v_{0}^{2}}{r} \\
N_{v_{0}}=-u_{0} \frac{\partial\left(r v_{0}\right)}{r \partial r}-w_{0} \frac{\partial v_{0}}{\partial z} ;
\end{gathered}
$$

and there is a similar term $N_{w_{0}}$ in the (non-hydrostatic) vertical momentum equation equal to minus the self-advection of $w_{0}$ by the wavenumber-zero disturbance flow. The vertical momentum equation also contains a term $F_{z}$ term analogous to $F_{r}$ in Equation (5), but with $u^{\prime}$ replaced by $w^{\prime}$ in the radial and vertical derivatives. However, we do not display these terms because the corresponding disturbances to hydrostatic balance were found to be relatively unimportant.

In a similar manner, the tendency equation for the wavenumber-zero disturbance potential temperature is given by

$$
\frac{\partial \theta_{0}}{\partial t}=-u_{0} \frac{\partial \Theta}{\partial r}-w_{0} \frac{\partial \Theta}{\partial z}+Q+N_{\theta_{0}} .
$$

The thermal forcing function $Q$ in Equation (9) is defined similarly to Equations (5) and (6) as follows:

$$
Q=\overline{\dot{\theta}}-\overline{u^{\prime} \frac{\partial \theta^{\prime}}{\partial r}}-\overline{v^{\prime} \frac{\partial \theta^{\prime}}{r \partial \lambda}}-\overline{w^{\prime} \frac{\partial \theta^{\prime}}{\partial z}}+\overline{S G_{\theta}}
$$

where $\dot{\theta}$ is the diabatic (radiative plus latent-heat) contribution to the material derivative of the potential temperature in the WRF model, and $S G_{\theta}$ denotes the heating rate due to subgrid-scale diffusion of potential temperature. In Equation (9), $N_{\theta_{0}}$ is the nonlinear term arising from the advection of the wavenumber-zero disturbance potential temperature by the wavenumber-zero disturbance flow and is defined as follows:

$$
N_{\theta_{0}}=-u_{0} \frac{\partial \theta_{0}}{\partial r}-w_{0} \frac{\partial \theta_{0}}{\partial z}
$$

When the quadratic terms $N_{u_{0}}, N_{v_{0}}, N_{w_{0}}$, and $N_{\theta_{0}}$ associated with the wavenumber-zero disturbance flow are small compared with the corresponding linear terms (defined by the first-order disturbance terms on the right-hand sides of Equations (1), (2) and (9)), it is justifiable to drop these quadratic terms in the foregoing equations. The 3DVPAS model for the wavenumber-zero disturbance flow is defined by the resulting linearized equations.

In their application of the 3DVPAS equations, Refs. [1,2] make three additional assumptions. We will make the same assumptions here so as not to introduce additional complexity when comparing 
with their published solutions: (1) in evaluating $V(r, z)$ from the azimuthally-averaged tangential wind in the WRF model, we take its value at $490 \mathrm{~m}$ altitude and extend that value downward so as to be independent of $z$ below $490 \mathrm{~m}$; (2) we assume a stress-free boundary condition on the disturbance horizontal velocity at $z=0$; (3) we neglect the $F_{r}$ forcing term, and its vertical counterpart $F_{z}$, keeping only $F_{\lambda}$ and $Q$, i.e., using only Equations (6) and (10).

Although we thus follow [1,2] in adopting their assumptions (1)-(3), we should point out before going further that we consider all three to be questionable. Assumption (1) is questionable because the condition $\partial V / \partial z=0$ at the lower boundary is inconsistent with the frictional, subgrid scale forcing diagnosed there from the WRF model, which is used to force the linear 3DVPAS model. Assumption (2) is questionable because the stress-free boundary condition implies that the disturbance flow at the lower boundary is subject to an artificial momentum source at the boundary. Finally, regarding assumption (3), although the neglect of $F_{z}$ is certainly acceptable for the system-scale vortex dynamics, recent work by [7] has shown that $F_{r}$ is, by contrast, not negligible-in fact comparable to $F_{\lambda}$ in the boundary layer, as is usual in rotating frictional boundary layers. Ref. [7] used a modestly high resolution cloud-permitting numerical simulation of an idealized tropical cyclone. We find the same to be true for the WRF model output used here (not shown). $F_{r}$ is comparable to $F_{\lambda}$ in the boundary layer because of the subgrid (frictional) contributions $\overline{S G}_{r}$ and $\overline{S G}_{\lambda}$ that tend to dominate there. Together with assumptions (1) and (2), the neglect of $F_{r}$ seriously distorts the boundary-layer dynamics by suppressing the frictionally-induced imbalance of the radial force field, which is an important component in producing strong low-level inflow.

We point out here further that Refs. [1,2] tacitly make an additional assumption by discarding the prime-prime eddy terms in Equations (6) and (10). Both Refs. [1,2] use the linear model with only the $S G$ terms retained in Equations (6) and (10) along with the $\bar{\theta}$ term in Equation (10). (From Ref. [1] (p. 2632): "The latent heating and the azimuthal component of friction from WRF are used to force 3DVPAS". From Ref. [2] (p. 1294): "The 12-h time-averaged WRF diabatic heating and PBL tangential wind tendency are shown in Figure 13c and 13d, respectively. The diabatic heating comes from direct model output from the WSM6 microphysics scheme, and the PBL term is the parameterized frictional tendency on tangential winds from the Yonsei University (YSU) scheme. We use these time-averaged tendencies as constant forcing terms for 3DVPAS".) However, since the WRF model is a fully three-dimensional model, and since three-dimensional eddy processes have been shown to be a non-negligible and important components of the intensification process of an idealized tropical cyclone [11], we prefer to retain the prime-prime eddy terms here.

For the calculations presented here, the forcing terms for the disturbance tangential velocity and diabatic heating (including the azimuthal average of the corresponding prime-prime eddy terms) are calculated from the WRF model output using the residual method:

$$
F_{\lambda}=\frac{\partial V}{\partial t}+\bar{\eta} U+W \frac{\partial V}{\partial z}
$$

and

$$
Q=\frac{\partial \Theta}{\partial t}+U \frac{\partial \Theta}{\partial r}+W \frac{\partial \Theta}{\partial z}
$$

Here, $(U, V, W, \Theta)$ denote the azimuthally-averaged radial, tangential and vertical velocity components, and the potential temperature, diagnosed from the WRF model output. By contrast with assumption (1) above, the symbol $V$ now temporarily includes the model's boundary-layer structure below $490 \mathrm{~m}$. These quantities and the differential operations in Equations (12) and (13) are evaluated at hour 27 in the WRF simulation, corresponding to $t=0$ in the 3DVPAS model. The time derivatives are computed as centered differences using data at hours 28 and 26. No formal accuracy is lost here by using the residual method because it has the same accuracy as a direct evaluation of $F_{\lambda}$ and $Q$ from Equations (5), (6) and (10). (To address a possible question about the potential inaccuracy of the residual method, for obtaining the partial time derivative terms using relatively coarse $(1 \mathrm{~h})$ model output, we have found that in Equations (12) and (13), the forcing terms $F_{\lambda}$ and $Q$ are dominated by 
the vertical and horizontal advection terms, and are relatively insensitive to the time derivatives and therefore still less sensitive to the precise time interval used to evaluate the finite differences. To assess the robustness of the residual method, $F_{\lambda}$ and $Q$ were calculated in two ways: the first calculation retained the advection terms, but discarded the time derivative terms altogether; the second calculation retained both the time derivative and the advection terms using the $1 \mathrm{~h}$ model output. The two results were found to be virtually identical.)

\section{Forcing the 3DVPAS Model}

The data used to force the 3DVPAS model are derived from a realistic WRF simulation of Hurricane Igor (2010). The storm reached tropical cyclone Category 4 in the Saffir-Simpson scale [20]. At the chosen time, hour 27, the storm was far less intense and in Category 1, but we will see that, even then, the nonlinearities were already becoming significant. Quadratic nonlinearities scale with the square of the wind speed, and so increase very steeply as Category 1 goes into Category 4.

The full-physics simulation was integrated with the Advanced Research core of the WRF model (ARW, [21]) version 3.2. The simulation was integrated by the Hurricane Group in the Mesoscale and Microscale Meteorology Division of the National Center for Atmospheric Research (NCAR). It is one of a set of model integrations that have been carried out since $2004[22,23]$. The simulation has three nested domains, with horizontal grid spacings of 12,4 and $1.33 \mathrm{~km}$ (with $469 \times 424,202 \times 202$, and $241 \times 241$ grid points, respectively), each of them with 35 vertical levels and each of them in a two-way nesting configuration. The outer domain is fixed and the two inner nests are centered at the location of the minimum $500 \mathrm{hPa}$ geopotential height, with repositioning performed every $15 \mathrm{~min}$. The Kain-Fritsch cumulus parameterization is used on the outer domain, but the inner domains have no cumulus parameterization since they run in cloud-permitting mode. The simulation uses the Yonsei University (YSU) scheme for the planetary boundary layer [24], a first-order closure scheme similar to the scheme of [25], but thought to be less biased towards excessive vertical mixing [26]. The surface drag algorithm follows [27]. The surface exchange coefficient for heat flux uses a similarity relationship [21] and that for water vapor follows [28]. The simulation was initialized at 12:00 a.m. UTC 11 September and integrated for $120 \mathrm{~h}$. At hour 27, having reached Category 1, the storm was only just becoming a tropical cyclone, and a coherent eyewall was imminent rather than fully formed.

Figure 1 shows the hourly evolution of maximum azimuthally averaged tangential wind velocity and minimum sea level pressure in the inner core of the WRF integration along with the corresponding 6-hourly National Hurricane Center best track information. The figure shows a relatively good performance of the simulation, particularly near hour 27 , the focus time of our analysis. The model integration captures the evolution of minimum sea level pressure particularly well in the first $40 \mathrm{~h}$ of integration. Within this period, the evolution of maximum wind velocity is relatively well captured also, after a gestation period during which the boundary layer becomes established and moistened by the surface moisture flux (not shown). The storm intensity evolution after hour 70 is largely influenced by the eyewall replacement cycle that Igor (2010) underwent. This notable structure, as well as the intensity change of Hurricane Igor, were well captured by the model integration (not shown) despite some discrepancies between the model integration and the Best Tracks dataset in terms of the variables shown in Figure 1.

To obtain the basic-state vortex fields, the azimuthal average pressure and density fields at hour 27 are used to derive $V$ as the corresponding gradient wind field from Equation (3), as in [29]. The resulting $V$ field is then smoothed with three-point running averages in the horizontal and in the vertical, applied twice each. (If the un-smoothed gradient wind is used to integrate the 3DVPAS model as explained in this manuscript, the model kinematic fields evolve unrealistically and absurdly large values are soon generated. In particular, the radial velocity exhibits values that surpass $50 \mathrm{~m} \mathrm{~s}^{-1}$ in the upper troposphere, within the first two hours of integration.) The $V$ field below $490 \mathrm{~m}$ is then altered to comply with assumption (1) below Equation (11). From the $V$ field thus altered, the balanced 
pressure, density and temperature fields, including a correspondingly altered $\Theta$ field, are obtained using Equations (3) and (4) and the methodology of [30].

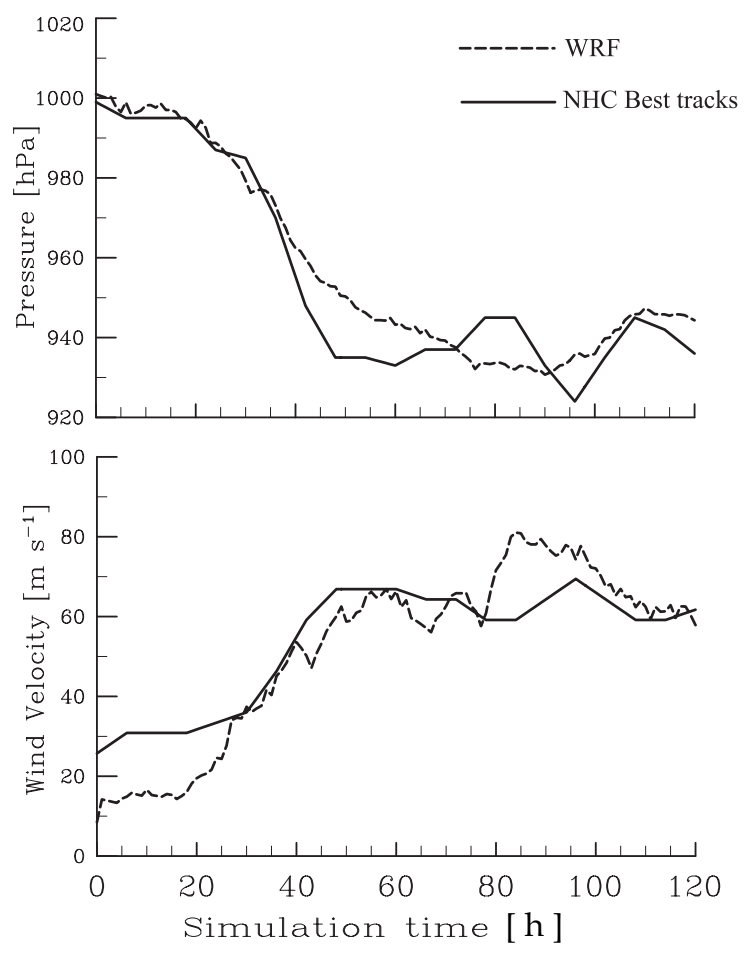

Figure 1. Maximum azimuthally averaged tangential wind velocity and minimum sea level pressure (hourly, dashed lines) in the inner core of the Weather Research and Forecasting (WRF) integration for Hurricane Igor (2010), and corresponding National Hurricane Center Best-Track information (6 hourly, solid lines).

\section{Results}

The time period around hour 27 is sufficient for illustrating the main point of this paper, i.e. the failure of the linear approximation even at this early stage of intensification. Similar analyses have been carried out for later times, including the time of the secondary eyewall formation period of Igor. As expected, they all show nonlinearities much stronger than those found below.

Figure 2 shows the basic-state $V$ and $\Theta$ fields and the corresponding forcing terms $Q$ and $F_{\lambda}$, as inferred from the WRF simulation at hour 27, but made consistent with assumption (1) as just explained. The $V$ and $\Theta$ fields are characteristic of a warm-cored cyclonic vortex in hydrostatic and gradient-wind balance. $V$ has a maximum of approximately $25 \mathrm{~m} \mathrm{~s}^{-1}$ near the lower surface and decreases monotonically with height, while the $\Theta$ isopleths curve downwards with decreasing radius. By itself, the $Q$ pattern (Figure 2c) suggests an annular eyewall with a localized heating pattern extending through the troposphere and sloping outwards. However, at this early stage of development, the convection exhibits a high degree of asymmetry and the $Q$ pattern comes from vertically coherent vortical plumes [11,31]. The $F_{\lambda}$ field (Figure $2 \mathrm{~d}$ ) exhibits a more complex pattern. First, as expected near the surface, there is a broad region out to a radius of about $60-70 \mathrm{~km}$ where $F_{\lambda}$ is largely negative. This reflects the near-surface friction felt by the tangential wind. Other prominent features are regions of strong positive and negative $F_{\lambda}$ within and near the incipient eyewall. Since $F_{\lambda}$ is computed as a residual, from Equation (12), it contains implicitly the rectified effect of eddy processes as noted above Equation (12). 

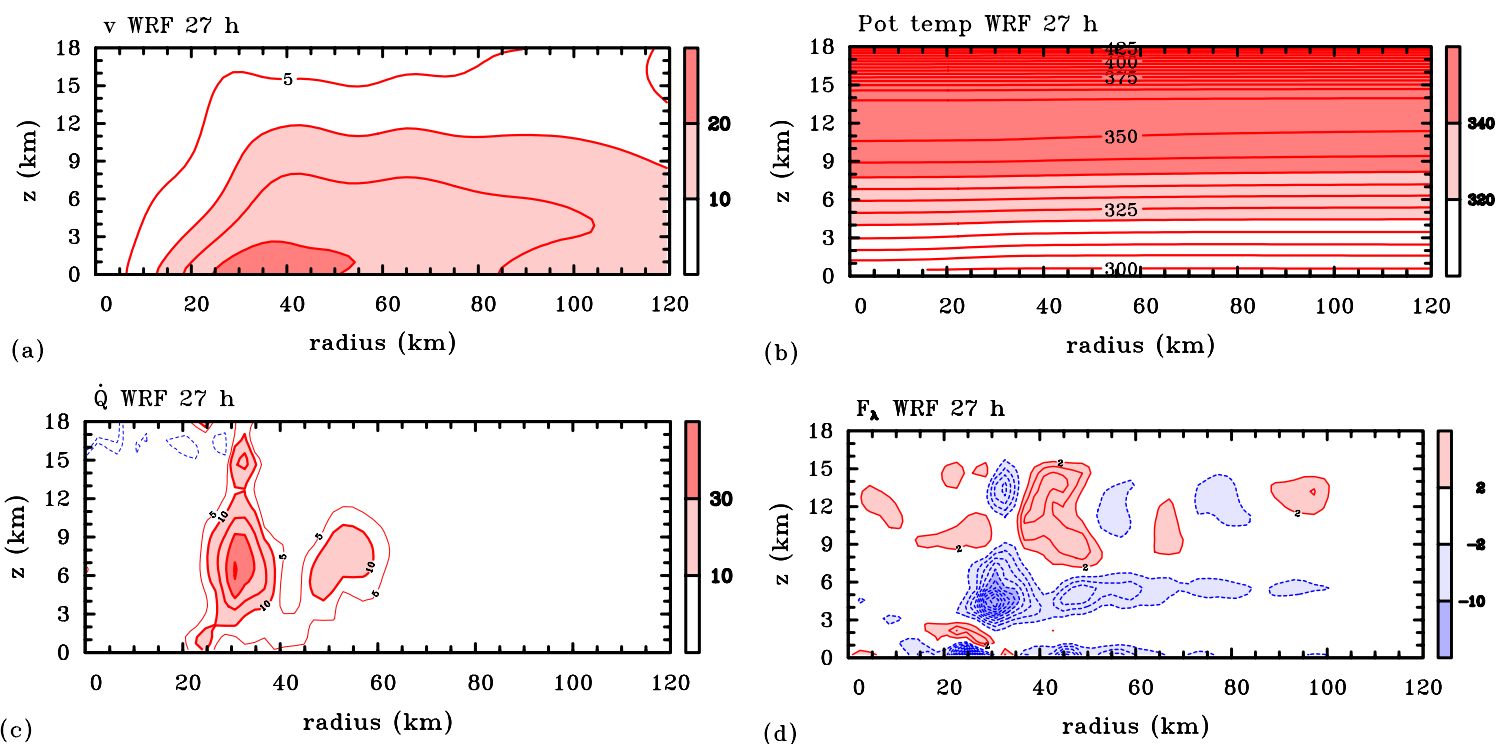

Figure 2. Calculated basic-state circular vortex and corresponding forcing terms as output from the WRF model at hour 27 in the simulation of the intensification of Hurricane Igor (2010). This basic state and these forcing terms (held constant) are used in the integration of the linear 3DVPAS model. Shown are the azimuthally-averaged (a) basic-state tangential velocity $V$ at hour 27 (contour interval $5 \mathrm{~m} \mathrm{~s}^{-1}$ ); (b) basic-state potential temperature $\Theta$ at hour 27 (contour interval $5 \mathrm{~K}$ ); (c) diabatic heating-rate forcing $\dot{Q}$ at hour 27 (thick contour interval is $10 \mathrm{~K} \mathrm{~h}^{-1}$, thin curve contour interval is $5 \mathrm{~K} \mathrm{~h}^{-1}$ ); and (d) tangential momentum forcing $F_{\lambda}$ at hour 27 (contour interval $2 \mathrm{~m} \mathrm{~s}^{-1} \mathrm{~h}^{-1}$, solid positive, dashed negative).

Figure 3 shows the $v_{0}, u_{0}$, and $w_{0}$ fields from the linear 3DVPAS model output at $t=8 \mathrm{~h}$, similar to integration times used in [1,2]. The $v_{0}$ pattern has positive values in the lower troposphere, just below the base of the $Q$ maximum in Figure 2c, and negative values within that maximum. The positive $v_{0}$ values at low levels are due primarily to the radial influx of basic-state vertical absolute vorticity, the linear term $-\bar{\eta} u_{0}$ in Equation (2). It dominates the other linear term $-w_{0} \partial V / \partial z$ as well as the forcing term $F_{\lambda}$, whose negative values are evident in Figure $2 \mathrm{~d}$. Earlier, at $t=0.4 \mathrm{~h}$, we have already seen positive values of $-\bar{\eta} u_{0}$ at low levels, as hinted in Figure 4 a below. Returning to Figure $3 \mathrm{a}$ and $t=8$ $\mathrm{h}$, we see that $v_{0}$ is negative somewhat higher in the troposphere. This is probably due primarily to the negative $F_{\lambda}$ values seen there in Figure $2 \mathrm{~d}$. In Figure $3 \mathrm{~b}$, the $u_{0}$ field shows a shallow layer of relatively strong inflow below $1 \mathrm{~km}$, and an upper-level outflow in the layer between about 11 and $15 \mathrm{~km}$. In Figure $3 c$, the $w_{0}$ structure near the incipient eyewall largely mimics the $Q$ structure seen in Figure $2 \mathrm{~d}$, with a primary maximum of $2.5 \mathrm{~m} \mathrm{~s}^{-1}$ at $r \approx 33 \mathrm{~km}$ and $z \approx 11 \mathrm{~km}$. The $w_{0}$ structure has a secondary maximum between $0.5 \mathrm{~m} \mathrm{~s}^{-1}$ and $1.0 \mathrm{~m} \mathrm{~s}^{-1}$ at $r \approx 55 \mathrm{~km}$ and $z \approx 10 \mathrm{~km}$ and a strong, broad minimum at $r \approx 83 \mathrm{~km}$ and $z \approx 9 \mathrm{~km}$.

Returning to the main point of this paper, we show now that, in this Hurricane Igor example, the 3DVPAS model becomes invalid, that is to say self-inconsistent, well before $t=8 \mathrm{~h}$. We show this by taking the 3DVPAS model output and using that output to evaluate some of the nonlinear terms that are neglected in the model. We then observe that these terms are not all uniformly small in magnitude by comparison with the terms that are retained, in defining the model and its response to the imposed forcing. 

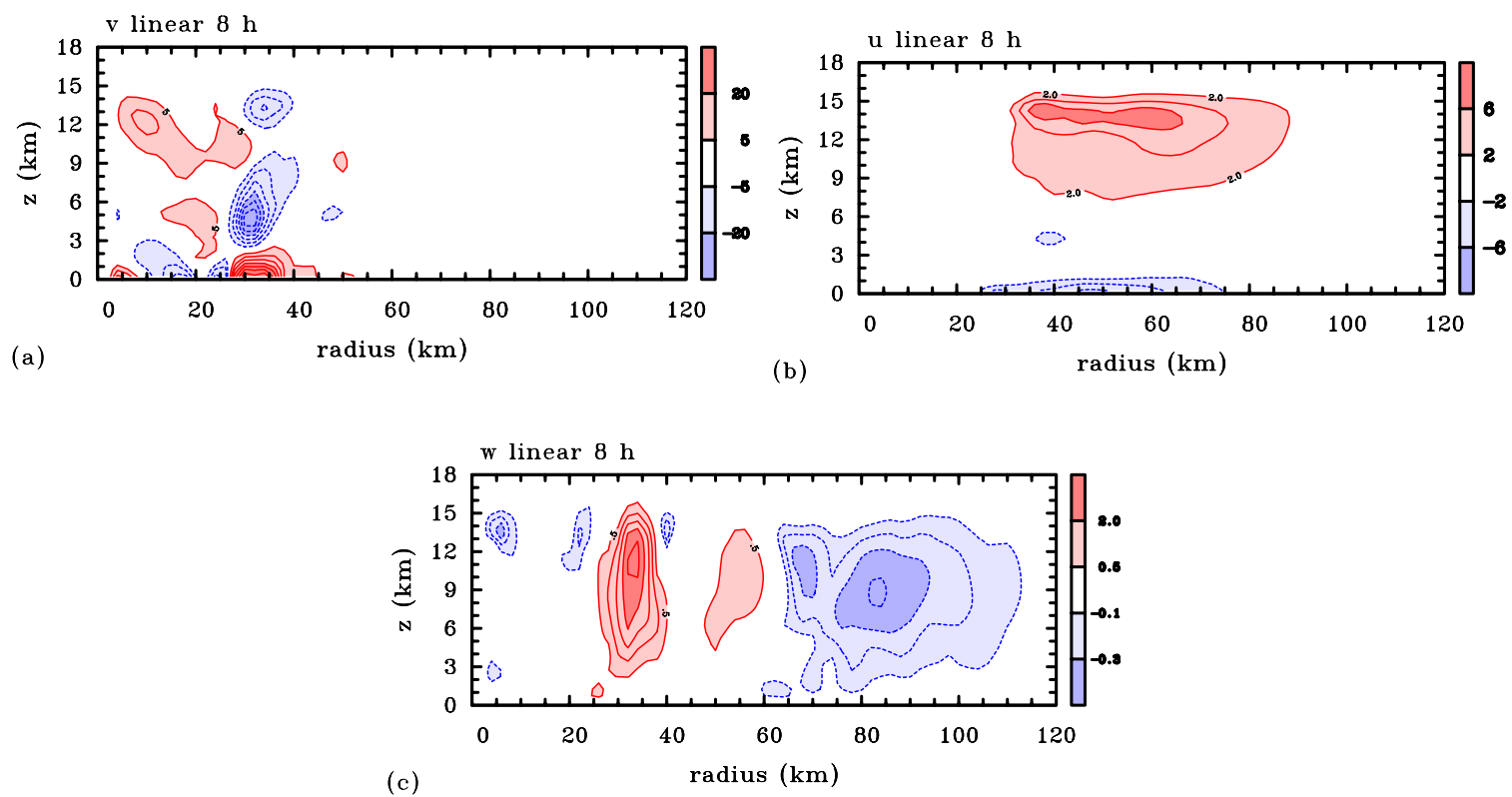

Figure 3. Output from the linear 3DVPAS model at $8 \mathrm{~h}$ integration time. Shown in a radius-height coordinate system are (a) tangential velocity disturbance $v_{0}$ (contour interval every $5 \mathrm{~m} \mathrm{~s}^{-1}$; dashed negative, solid positive); (b) radial velocity disturbance $u_{0}$ (contour interval $2 \mathrm{~m} \mathrm{~s}^{-1}$; dashed negative, solid positive); (c) vertical velocity disturbance $w_{0}$ (positive contour interval $0.5 \mathrm{~m} \mathrm{~s}^{-1}$, solid; negative contour interval $0.1 \mathrm{~m} \mathrm{~s}^{-1}$, dashed).

We focus attention on Equations (1) and (2), in which the tendencies or rates of change as the model responds to the imposed forcing are determined entirely by the forcing terms together with the linear terms. The linear terms are the first pair of terms on each right-hand side of Equations (1) and (2), whose sums will be referred to as the " $u$ linear tendency" and the " $v$ linear tendency", respectively. Figures 4 and 5 compare those linear tendencies (panels on the left) with the the nonlinear terms $N_{u_{0}}$ and $N_{v_{0}}$ neglected in the model (panels on the right). The neglected terms, evaluated from the linear solution, will be referred to as the " $u$ nonlinear tendency" and the " $v$ nonlinear tendency", respectively.

Even at the very early time $t=0.4 \mathrm{~h}$ (Figure 4 ), there are parts of the domain in which the nonlinear tendencies are, conspicuously, not much smaller than the linear tendencies, indeed comparable or greater in magnitude, most notably in the region of the incipient eyewall. A little later, at $t=1.2 \mathrm{~h}$ (Figure 5), the nonlinear tendencies are very much bigger in some places. This is more than enough to support our conclusion that, in this example, the 3DVPAS model has predicted its own breakdown and has become self-inconsistent, and therefore invalid. Still less is the model valid at $t=8 \mathrm{~h}$. If the analysis is repeated, but with forcing terms derived from the WRF model at times closer to full intensity, then we find, not surprisingly, that the magnitudes of the nonlinear terms have increased very steeply indeed.

At $t=1.2 \mathrm{~h}$, there are already hints in Figure $5 \mathrm{~d}$ of what can be seen much more clearly in Ref. [7], namely the emergence of strong, non-Ekman-like nonlinearities near the base of the eyewall at the later stages of intensification, giving rise to strong supergradient winds, much stronger than those obtained from Ekman-like dynamics. This is the signature of the nonlinear spinup mechanism, which significantly increases the destructive power of a tropical cyclone. The signature becomes very clear as Igor approaches full intensity. 

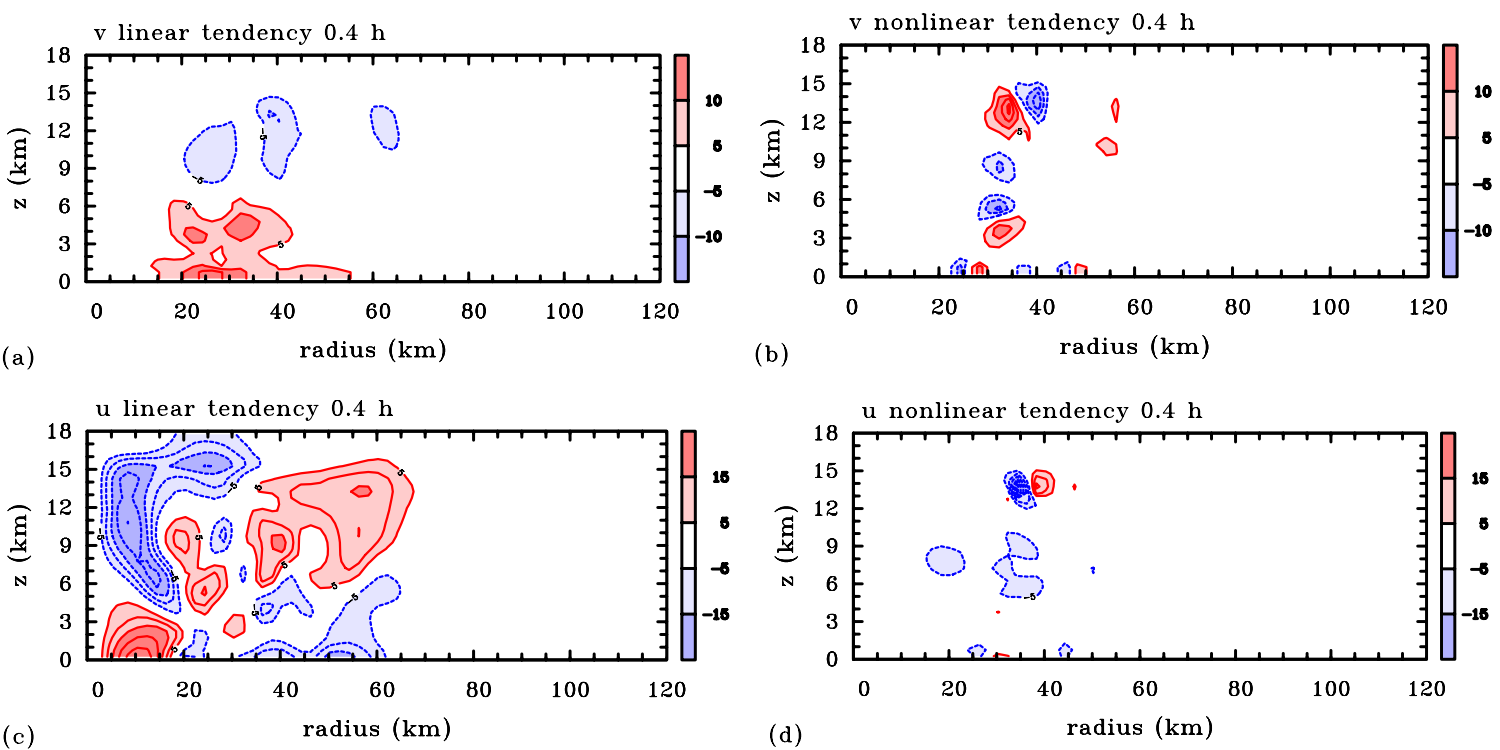

Figure 4. Linear tendency terms diagnosed from the 3DVPAS model and corresponding nonlinear tendency terms evaluated using the linear solutions at $t=0.4 \mathrm{~h}$ integration time. Shown in a radius-height coordinate system are (a) linear terms from the disturbance tangential velocity equation; (b) nonlinear terms in the disturbance tangential velocity equation (Equation (8)) evaluated using the linear solutions; (c) linear terms in disturbance radial velocity equation; (d) nonlinear terms in disturbance radial velocity equation (Equation (7)) computed using linear solutions. Contour interval for all panels is $5 \mathrm{~m} \mathrm{~s}^{-1} \mathrm{~h}^{-1}$, solid positive and dashed negative.
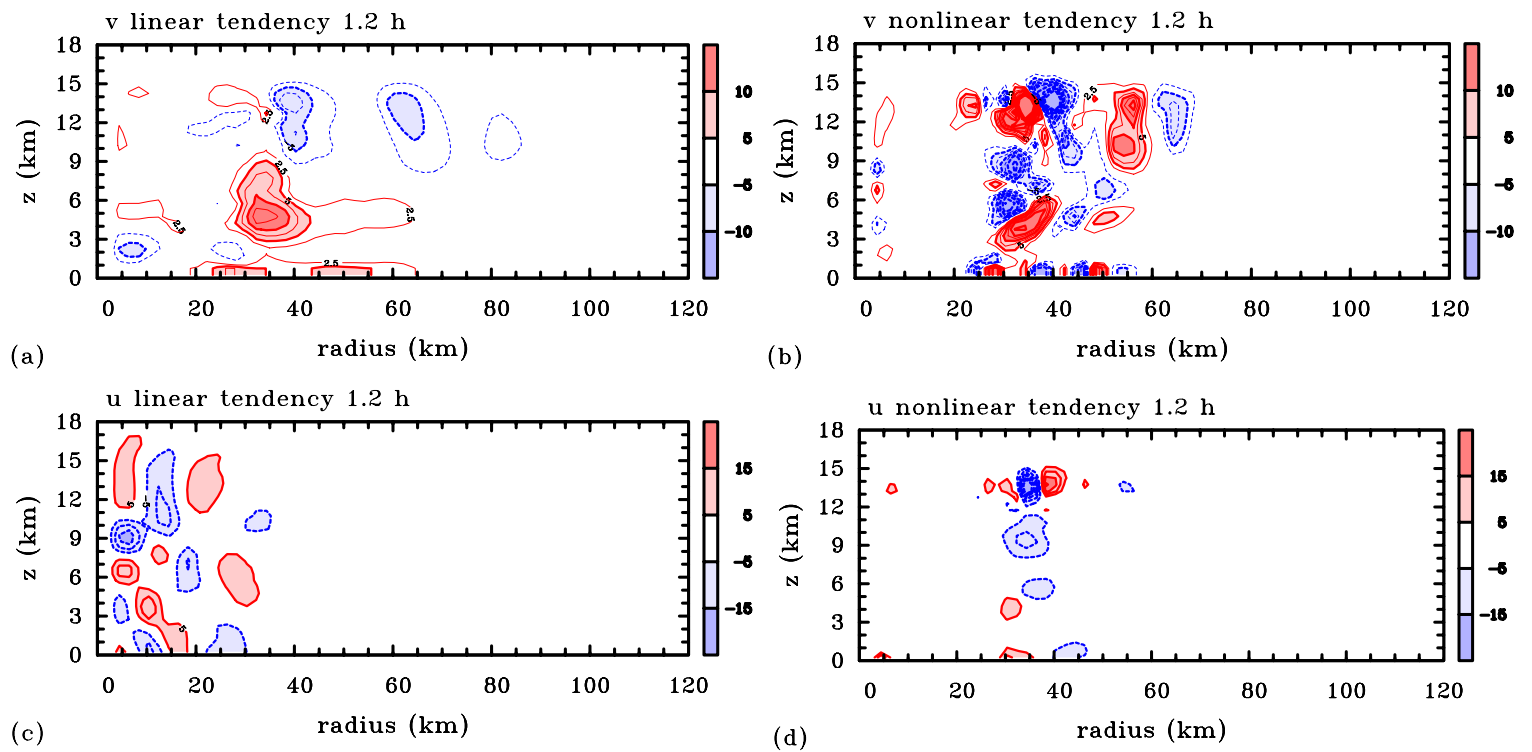

Figure 5. Linear and nonlinear tendency terms diagnosed from the 3DVPAS model at $t=1.2 \mathrm{~h}$ integration time. The nonlinear tendency terms are evaluated using the linear solutions. Shown in a radius-height coordinate system are (a) sum of linear terms from the disturbance tangential velocity equation; (b) sum of nonlinear terms in the disturbance tangential velocity equation (Equation (8)) evaluated using the linear solutions; (c) sum of linear terms in disturbance radial velocity equation; (d) sum of nonlinear terms in disturbance radial velocity equation (Equation (7)) computed using linear solutions. Contour interval for all panels is $2 \mathrm{~m} \mathrm{~s}^{-1} \mathrm{~h}^{-1}$; solid positive and dashed negative. 


\section{3DVPAS Dynamics, Sawyer-Eliassen Balance Dynamics, and Their Possible Extensions}

Refs. $[1,2,17,18]$ bring up the issue of 3DVPAS dynamics versus the dynamics of the traditional Sawyer-Eliassen (SE) model of transverse circulations. Since both are linearized models in the sense already discussed, the only differences are those arising from the unbalanced dynamics allowed by 3DVPAS versus the strictly balanced dynamics built in to SE. The SE model, as is well known, incorporates hydrostatic and gradient-wind balance from the start.

If, for instance, 3DVPAS is run with steady forcing terms, as above, and we wait for long enough after the forcing terms are switched on $(t \gg 0.4 \mathrm{~h}$, typically between 6 and $12 \mathrm{~h}$, in examples like the foregoing), then the 3DVPAS behavior approaches that of the SE model. Somewhat confusingly, Ref. [17] calls this long-time behaviour "quasi-steady", even though the tangential wind and potential temperature are still changing like $t$, in the well known way. It is not, of course, essential to use steady forcing terms. More generally, as discussed below, we may expect 3DVPAS behavior to resemble SE behavior whenever the forcing terms vary slowly enough in time.

Being a statement about the comparative behaviors of two linearized models, the foregoing is entirely separate from our main point, that the linearization itself becomes invalid at comparatively short times $t \sim 0.4 \mathrm{~h}$ in the context of maturing and mature tropical cyclones, especially as regards the inner-core boundary layer. It is of interest, nevertheless, to ask whether in that context either model could be rendered useful by restoring the neglected nonlinear $N$ terms. The rest of this section will tentatively conclude that both models could indeed become useful in this way, although the numerical experiments needed to examine the question more closely are beyond the scope of this paper and will be left for future work.

We proceed as follows. Starting within the framework of the unbalanced 3DVPAS equations, we derive an extended SE equation that retains all forcing terms in the disturbance equations of Section 2, as well as the nonlinear $N$ terms in the momentum equations and in the corresponding thermodynamic equation. Retention of the radial as well as the tangential horizontal forcing terms is consistent with the findings of the previous section. We retain also the time derivatives that are usually neglected, but then discuss which of these should be included in order to make the equation as useful as possible in the current context.

For simplicity, we adopt the Boussineq approximation wherein density disturbances are retained only when multiplied by the gravitational acceleration, but neglected elsewhere (e.g., [29], Ch.7). The generalization to the vortex-anelastic equations is straightforward. The reference density with disturbances neglected will be denoted by $\rho_{a}$, a constant. The disturbance continuity equation is that of incompressible flow,

$$
\frac{\partial\left(r u_{0}\right)}{\partial r}+\frac{\partial\left(r w_{0}\right)}{\partial z}=0
$$

and the disturbance vertical momentum equation is

$$
\frac{\partial w_{0}}{\partial t}=-\frac{1}{\rho_{a}} \frac{\partial p_{0}}{\partial z}+b_{0}+\tilde{F}_{z}
$$

where $\tilde{F}_{z}$ is shorthand for the sum $F_{z}+N_{w_{0}}$ referred to below in Equation (8), and where $b_{0}$ satisfies the Boussinesq counterpart of Equation (9),

$$
\frac{\partial b_{0}}{\partial t}=-u_{0} \frac{\partial B}{\partial r}-w_{0} \frac{\partial B}{\partial z}+\tilde{Q} .
$$

Here, $B(r, z)$ and $b_{0}(r, z, t)$ are the background and disturbance Boussinesq buoyancy accelerations, and $\tilde{Q}$ is shorthand for the sum of the last two terms in Equation (9), i.e., the thermal forcing term $Q$ and the nonlinear term $N_{\theta_{0}}$, or rather their Boussinesq counterparts. $B$ and $b_{0}$ are defined in the standard way as gravitational acceleration times fractional density anomaly. To complete the system, we use the Boussinesq forms of Equations (1) and (2), which are unaltered apart from replacing $\rho$ by $\rho_{a}$ in Equation (1) and introducing the shorthand notations $\tilde{F}_{r}=F_{r}+N_{u_{0}}$ and $\tilde{F_{\lambda}}=F_{\lambda}+N_{v_{0}}$. 
Now we can derive a single equation for the transverse circulation $\left(u_{0}, w_{0}\right)$ by subtracting $\partial^{2} / \partial r \partial t$ from Equation (15) from $\partial^{2} / \partial z \partial t$ from Equation (1), and then eliminating $\partial v_{0} / \partial t$ and $\partial b_{0} / \partial t$ using Equations (2) and (16), respectively. The result is

$$
\begin{aligned}
\frac{\partial}{\partial z}\left[\left(\frac{\partial^{2}}{\partial t^{2}}+\bar{\xi} \bar{\eta}\right) u_{0}\right]-\frac{\partial}{\partial r}\left[\left(\frac{\partial^{2}}{\partial t^{2}}+\frac{\partial B}{\partial z}\right) w_{0}\right] \\
+\frac{\partial}{\partial z}\left(\bar{\xi} \frac{\partial V}{\partial z} w_{0}\right)-\frac{\partial}{\partial r}\left(\frac{\partial B}{\partial r} u_{0}\right)=-\frac{\partial \tilde{Q}}{\partial r}+\frac{\partial}{\partial z}\left(\bar{\xi} \tilde{F_{\lambda}}\right) \\
+\frac{\partial^{2} \tilde{F}_{r}}{\partial z \partial t}-\frac{\partial^{2} \tilde{F}_{z}}{\partial r \partial t} .
\end{aligned}
$$

As defined in Section 2, upper-case $V$ denotes the azimuthally-averaged tangential velocity of the circular vortex and $(\bar{\eta}, \bar{\xi})$ denotes the local absolute vertical vorticity and twice the absolute rotation rate of the circular vortex flow, respectively.

When a streamfunction $\psi$ is introduced for the transverse circulation such that Equation (14) is satisfied, i.e., $u_{0}=-(1 / r) \partial \psi / \partial z$ and $w_{0}=(1 / r) \partial \psi / \partial r$, then a single partial differential equation results for $\psi$. The resulting equation (not written) is analogous to the single streamfunction equation derived by Hoskins [32] in his pioneering study of the symmetric stability and instability of middle-latitude fronts. Notice that if $\tilde{F}_{r}, \tilde{F}_{\lambda}, \tilde{F}_{z}$ and $\tilde{Q}$ and the response described by (17) are all assumed to vary sufficiently slowly, then we may neglect all the time derivatives in Equation (17), giving us the standard SE equation, in which balance allows us to replace $\partial B / \partial r$ by $\bar{\xi} \partial V / \partial z$.

More subtly, however, we may consistently keep the first time derivatives on the right-hand side of Equation (17), while neglecting the second time derivatives on the left-hand side, if rates of variation are "fairly slow" in the following sense. To neglect the second derivatives, we need $\partial^{2} / \partial t^{2}$ to be small in comparison with $\bar{\xi} \bar{\eta}$ and $\partial B / \partial z$, the squares of the local inertial frequency and buoyancy frequency. There is a range of conditions for which the first derivatives can nevertheless remain significant, especially in the first term on the third line of Equation (17), whose magnitude in comparison with the last term on the middle line can be significant. On the reasonable assumption that $F_{r} \sim F_{\lambda}$, we need to compare $\partial / \partial t$ with $\bar{\xi}$, which is more like the square root of the local inertial frequency. In our judgment, such "fairly slow" evolution is often relevant to tropical cyclone evolution ([33]). We then have

$$
\frac{\partial}{\partial r}\left[\frac{\partial B}{\partial z} \frac{1}{r} \frac{\partial \psi}{\partial r}-\frac{\partial B}{\partial r} \frac{1}{r} \frac{\partial \psi}{\partial z}\right]+\frac{\partial}{\partial z}\left[\frac{\bar{\xi} \bar{\eta}}{r} \frac{\partial \psi}{\partial z}-\frac{\bar{\xi}}{r} \frac{\partial V}{\partial z} \frac{\partial \psi}{\partial r}\right]=\frac{\partial \tilde{Q}}{\partial r}-\frac{\partial}{\partial z}\left(\bar{\xi} \tilde{F}_{\lambda}\right)-\frac{\partial^{2} \tilde{F}_{r}}{\partial z \partial t}+\frac{\partial^{2} \tilde{F}_{z}}{\partial r \partial t} .
$$

This has diagnostic form, like the standard SE equation, if the right-hand side can be regarded as known. It will therefore be called an extended SE equation. However, it should be remembered that the nonlinear $N$ terms on the right then need to be treated iteratively. It needs also to be checked whether the partial differential operator on the left is elliptic. Ellipticity can fail in the inner-core boundary layer under nonlinear spinup conditions, which may be one of the reasons why Refs. [1,2] made their assumptions (1)-(3).

Equation (18) is elliptic if the vortex is symmetrically stable (i.e., if the inertial stability on isentropic surfaces is greater than zero); and symmetric stability is assured [33] when $\bar{\eta} \bar{\xi}(\partial B / \partial z)-(\bar{\xi}(\partial V / \partial z))^{2}>$ 0 , assuming that we can use the balance relation $\partial B / \partial r=\bar{\xi} \partial V / \partial z$ for this purpose.

The extended SE Equation (18) can be used to describe at least some of the effects of nonlinear boundary-layer spinup, via iterative correction. That is, the $N$ terms included on the right can be evaluated from a linear solution, then the equation solved again, the $N$ terms evaluated more accurately, and so on. This iterative procedure corresponds to that used in the classic idealized studies by Kuo [34,35], in which an Ekman-like boundary layer model was iteratively corrected for the non-Ekman-like nonlinearities, verifying that these produced significant strengthening of the supergradient winds near the top of the boundary layer. However, such an iterative procedure cannot describe the sort of drastic breakdown of balance that can occur as the boundary layer undergoes separation and turns upward into an eyewall. 
It is here that Equation (17), the fully-unbalanced counterpart of (18), could become useful. At this level of description, with all the time derivatives included, we would have something equivalent to 3DVPAS dynamics rather than to a somewhat-extended SE dynamics. Although this remains to be verified-a topic for future work-it is possible that we could then begin to describe the emission of freely-propagating inertia-gravity waves from the separating boundary layer into the eyewall.

\section{Conclusions}

We have re-examined the utility of an unbalanced axisymmetric convective ring model using the dry, non-hydrostatic, time dependent linear vortex model 3DVPAS. This model was developed originally to examine linear asymmetric disturbances and their wave-mean-flow interactions in tropical cyclones and tornado vortices, and has produced many insightful results for that class of problems. Recent work [1,2] has used the 3DVPAS model in a different way, to study intensifying tropical cyclones by solving for the azimuthal-wavenumber-zero disturbance flow and corresponding tangential wind tendency subject to the imposed forcing of heat and tangential momentum sources. Using diabatic heating, tangential friction, and eddy forcing terms derived from a three-dimensional numerical simulation of an intensifying tropical cyclone vortex, Hurricane Igor, we have again investigated this different way of using of the 3DVPAS model, but this time with attention to whether, in this context, it is valid to neglect the nonlinear effects omitted from the model, the $N$ terms in the equations described in Section 2.

We found that the linear 3DVPAS solutions quickly break down in the sense that the neglected $N$ terms, calculated from the output of the linear model, become comparable to or larger than the linear terms in the governing momentum equations. This breakdown occurs on a time scale given approximately by the time required to develop the vortex boundary layer and incipient eyewall in the 3DVPAS model. The breakdown of the linear solution is found to be particularly severe in the inner-core boundary layer and in the eyewall of the simulated vortex.

The demonstrated breakdown of linearity supports the scientific arguments made in [6] that (a) non-Ekman-like nonlinearities and nonlinear spinup are important in the inner-core boundary layer of an intensifying tropical cyclone and are responsible for the realistic growth of peak supergradient winds, and that (b) it is not valid to use a linearized model to isolate the separate effects of diabatic heating from those of friction. This is simply because the linear superposition principle breaks down whenever the dynamics are not linear.

Further to our second objective regarding the traditional Sawyer-Eliassen (SE) model, we have shown how an extended SE Equation (18) may be derived from the 3DVPAS model formulation, the latter corresponding to Equation (17) above, with the addition of the nonlinear $N$ terms and also the radial and vertical momentum source terms associated with vertical subgrid scale mixing associated with boundary layer processes that have been neglected in prior applications.

The traditional SE model requires that the forcing terms vary sufficiently slowly with time, a requirement that is typically quite severe in a tropical cyclone undergoing rapid intensification. For this reason, our extended SE Equation (18) also includes the first time derivatives shown on its right-hand side, which allows somewhat faster variation with time, discussed in Section 5 as "fairly slow" variation. For still faster variation and fully unbalanced motion, it is necessary to retain also a pair of second time derivatives, those on the left of Equation (17), which restores the level of modeling to that of 3DVPAS.

It would be interesting in future work to make use of Equations (17) and (18), and their vortex-anelastic counterparts, in an approach to modeling and better understanding the nonlinear effects in intensifying tropical cyclones. The first stage would be to evaluate the nonlinear $N$ terms from the linear solutions, as in Section 4, then solve Equations (17) or (18) again, then repeat the process to make iterative corrections that represent the effects of the $N$ terms with increasing accuracy. Such an iterative process would correspond to that explored in the classic work of Kuo [34,35] on 
non-Ekman-like boundary-layer nonlinearities, and promises to yield a sharpened understanding of the growth of supergradient winds.

Acknowledgments: The authors wish to thank Professor Dave Nolan for providing the current version of the 3DVPAS model to us in December 2014. The authors also thank Sergio Abarca for his expertise with the Igor WRF data and set up and execution of the 3DVPAS model. Finally, the authors thank Professor Michael E. McIntyre at the University of Cambridge and three anonymous reviewers for their perceptive comments on the original manuscript. M.T.M. acknowledges the support of National Science Foundation (NSF) AGS-1313948, National Oceanic and Atmospheric Administration (NOAA) Hurricane Forecast Improvement Project (HFIP) grant N0017315WR00048, National Aeronautics and Space Administration (NASA) grant NNG11PK021, ONR N0001417WX00336 and the U.S. Naval Postgraduate School. R.K.S. acknowledges financial support for this research from the German Research Council (Deutsche Forschungsgemeinschaft) under Grant No. SM30-23 and the Office of Naval Research Global under Grant No. N62909-15-1-N021. The views expressed herein are those of the authors and do not represent sponsoring agencies or institutions.

Author Contributions: Michael T. Montgomery and Roger K. Smith conceived this work; Sergio Abarca set up and executed experiments; Michael T. Montgomery, Roger K. Smith and Sergio Abarca analyzed the data; Michael T. Montgomery and Roger K. Smith wrote the paper.

Conflicts of Interest: The authors declare no conflict of interest.

\section{References}

1. Rozoff, C.M.; Nolan, D.S.; Kossin, J.P.; Zhang, F.; Fang, J. The roles of an expanding wind field and inertial stability in tropical cyclone secondary eyewall formation. J. Atmos. Sci. 2012, 69, 2621-2643.

2. Stern, D.P.; Vigh, J.L.; Nolan, D.S.; Zhang, F. Revisiting the relationship between eyewall contraction and intensification. J. Atmos. Sci. 2015, 72, 1283-1306.

3. Willoughby, H.E. Temporal changes of the primary circulation in tropical cyclones. J. Atmos. Sci. 1990, 47, 242-264.

4. Willoughby, H.E. Mature Structure and Evolution; WMO/TD- No 693; Elsberry, R.L., Ed.; World Meteorological Organization: Geneva, Switzerland, 1995; p. 289.

5. Vogl, S.; Smith, R.K. Limitations of a linear model for the hurricane boundary layer. Q. J. R. Meteorol. Soc. 2009, 135, 839-850.

6. Smith, R.K.; Montgomery, M.T. Towards clarity on tropical cyclone intensification. J. Atmos. Sci. 2015, 72, doi:10.1175/JAS-D-15-0017.1.

7. Abarca, S.F.; Montgomery, M.T.; McWilliams, J.C. The azimuthally-averaged boundary layer structure of a numerically simulated major hurricane. J. Adv. Model. Earth Syst. 2015, 7, 1207-1219.

8. Smith, R.K.; Montgomery, M.T. Hurricane boundary-layer theory. Q. J. R. Meteorol. Soc. 2010, 136, $1665-1670$.

9. Montgomery, M.T.; Smith, R.K. Paradigms for tropical cyclone intensification. Aust. Meteorol. Oceanogr. J. 2014, 64, 37-66.

10. Rotunno, R. Secondary circulations in rotating-flow boundary layers. Aust. Meteorol. Oceanogr. J. 2014, 64, 27-35.

11. Persing, J.; Montgomery, M.T.; McWilliams, J.; Smith, R.K. Asymmetric and axisymmetric dynamics of tropical cyclones. Atmos. Chem. Phys. 2013, 13, 12299-12341.

12. Nolan, D.S.; Montgomery, M.T. Three-dimensional, nonhydrostatic perturbations to balanced, hurricane-like vortices. Part I: Linearized formulation, stability, and evolution. J. Atmos. Sci. 2002, 59, 2989-3020.

13. Rotunno, R. The fluid dynamics of tornadoes. Annu. Rev. Fluid Mech. 2013, 45, 59-84.

14. Nolan, D.S. Three dimensional instabilities in tornado like vortices with secondary circulations. J. Fluid Mech. 2012, 711, 61-100.

15. Moon, Y.; Nolan, D.S. Do gravity waves transport angular momentum away from tropical cyclones? J. Atmos. Sci. 2010, 67, 117-135.

16. Hodyss, D.; Nolan, D.S. The Rossby-Inertia-Buoyancy instability in baroclinic vortices. Phys. Fluids 2008, 20, 096602.

17. Nolan, D.S.; Grasso, L.D. Nonhydrostatic, three-dimensional perturbations to balanced, hurricane-like vortices. Part II: Symmetric response and nonlinear simulations. J. Atmos. Sci. 2003, 60, 2717-2745.

18. Hodyss, D.; Nolan, D.S. Linear anelastic equations for atmospheric vortices. J. Atmos. Sci. 2007, 64, $2947-2959$. 
19. Nolan, D.S.; Moon, Y.; Stern, D.P. Tropical cyclone intensification from asymmetric convection: Energetics and efficiency. J. Atmos. Sci. 2007, 64, 3377-3405.

20. Pasch, R.J.; Kimberlain, T.B. National Hurricane Center, NOAA, 2011. Available online: http://www.nhc. noaa.gov/data/tcr/AL112010_Igor.pdf (accessed on 30 November 2017).

21. Skamarock, W.C.; Klemp, J.B.; Dudhia, J.; Gill, D.O.; Barker, D.M.; Wang, W.; Powers, J.G. A description of the Advanced Research WRF version 2. NCAR Tech. Note 2005, doi:10.5065/D6DZ069T.

22. Davis, C.; Wang, W.; Chen, S.S.; Chen, Y.; Corbosiero, K.; DeMaria, M.; Dudhia, J.; Holland, G.; Klemp, J.; Michalakes, J.; et al. Prediction of landfalling hurricanes with the advanced hurricane WRF model. Weather Forecast. 2008, 119, 1826-1841.

23. Davis, C.; Wang, W.; Dudhia, J.; Torn, R. Does increased horizontal resolution improve hurricane wind forecasts? Weather Forecast. 2010, 119, 1826-1841.

24. Noh, Y.; Cheon, W.G.; Hong, S.Y.; Raasch, S. Improvement of the K-profile model for the planetary boundary layer based on large eddy simulation data. Bound. Layer Meteorol. 2003, 107, 421-427.

25. Hong, S.Y.; Pan, H.L. Nocturnal boundary layer vertical diffusion in a medium-range forecast model. Mon. Weather Rev. 1996, 124, 2322-2339.

26. Braun, S.A.; Tao, W.K. Sensitivity of high-resolution simulations of hurricane Bob (1991) to planetary boundary layer parameterizations. Mon. Weather Rev. 2000, 128, 3941-3961.

27. Charnock, H. Wind stress on a water surface. Q. J. R. Meteorol. Soc. 1995, 81, 639-640.

28. Carlson, T.N.; Boland, F.E. Analysis of urban-rural canopy using a surface heat flux/temperature model. J. Appl. Meteorol. Climatol. 1978, 17, 998-1013.

29. Holton, J.R. An Introduction to Dynamic Meteorology; Elsevier Academic Press: Burlington, MA, USA, 2004; p. 535.

30. Smith, R.K. Accurate determination of a balanced axisymmetric vortex. Tellus 2006, 58, 98-103.

31. Smith, R.K.; Kilroy, G.; Montgomery, M.T. Why do model tropical cyclones intensify more rapidly at low latitudes? J. Atmos. Sci. 2015, 72, 1783-1804.

32. Hoskins, B.J. The role of potential vorticity in symmetric stability and instability. Q. J. R. Meteorol. Soc. 1975, 100, 480-482.

33. Shapiro, L.J.; Montgomery, M.T. A three-dimensional balance theory for rapidly-rotating vortices. J. Atmos. Sci. 1993, 50, 3322-3335.

34. Kuo, H.L. Axisymmetric flows in the boundary layer of a maintained vortex. J. Atmos. Sci. 1971, $28,20-41$.

35. Kuo, H.L. Vortex boundary layer under quadratic surface stress. Bound. Layer Meteorol. 1982, 22, 151-169. 